\title{
Response to:"Medical Student Perspectives on Their Role as Emerging Physicians During the COVID-19 Pandemic"
}

\author{
Suhaylah Adam ${ }^{1} \mathbb{D} \cdot$ Celina J. Pook ${ }^{1} \cdot$ Tabea Haas-Heger $^{1} \cdot$ Sophie Simmonds ${ }^{1} \cdot$ Molly Kirkman $^{1}$
}

Accepted: 19 November 2021 / Published online: 1 December 2021

(c) The Author(s) under exclusive licence to International Association of Medical Science Educators 2021

\begin{abstract}
We present a Letter to the Editor in response to the article by Chinsky et al. titled "Medical Student Perspectives on Their Role as Emerging Physicians During the COVID-19 Pandemic." The authors highlighted that a greater proportion of preclinical students felt more obligated to volunteer in the context of healthcare staff shortages, compared to clinical students. As final year medical students, we propose additional reasons based on our personal experiences that may contribute to clinical students feeling less inclined to volunteer during the pandemic. Specifically, we believe that the priorities of clinical students may affect their willingness to volunteer given the challenge of balancing private study and clinical placement. Furthermore, we believe that the psychological difficulties that often accompany clinical placement may affect clinical students' views on volunteering. We also briefly outline solutions that may solve these issues. Ultimately, positive attitudes in students need to be sustained throughout the medical degree so that the future generation of doctors is better equipped to face a future crisis and beyond.
\end{abstract}

Keywords Medical education $\cdot$ COVID-19 $\cdot$ Student perspectives $\cdot$ Volunteering

\section{Dear Editor,}

We read with great interest the article by Chinsky et al. [1], which elicited medical student perspectives regarding their role during the COVID-19 pandemic. The authors highlighted that preclinical students felt more obligated to volunteer in the context of healthcare staff shortages, compared to clinical students. As final year medical students, we propose additional reasons based on our experiences that may contribute to clinical students feeling less inclined to volunteer, whilst also outlining strategies that may sustain students' willingness to enrol in the future.

Whilst we agree that clinical students may feel more apprehensive to volunteer given their exposure to the rapidly deteriorating clinical environment, we believe that their priorities may also influence their perspective. Specifically, clinical students face a significant challenge in balancing private study and clinical placement in comparison to preclinical students. Indeed, research has identified a greater

Suhaylah Adam

suhaylah.adam@kcl.ac.uk

1 GKT School of Medical Education, King's College London, Newcomen Street, SE1 1UL London, UK prevalence of burnout during clinical years [2]. Concurrent responsibilities alongside emotional exhaustion may lead to a conflict in values [3], which can cloud students' ethical judgement on joining the frontline. Additionally, clinical students may develop a greater awareness of the importance of a work-life balance as they advance towards securing their first jobs, prompting them to reconsider their priorities. As such, we believe that students require support in managing their time. For example, compulsory workshops in which tutors outline personal approaches to task prioritisation would help students to become better equipped in dealing with additional responsibilities.

The psychological challenges that often accompany clinical education may also influence clinical students' readiness to volunteer [3]. At our university, clinical students frequently rotate hospitals in contrast to preclinical students. Given that geographical isolation may affect wellbeing in clinical students [4], individuals may need to use their free time to prioritise self-care over serving during the pandemic. Additionally, distress caused by an emotionally challenging clinical environment may compromise students' moral stance on volunteering. Balint groups may offer a pertinent solution to these issues; reflecting on the psychological aspects of patient encounters may enable students to resolve 
internal conflict [5]. Moreover, group discussion may foster a sense of community, thereby mitigating loneliness.

Clinical students experience a range of challenges. As such, it is crucial that their perspectives are thoroughly elicited so that solutions can be implemented. By sustaining positive attitudes in students, the future generation of doctors will be better prepared to volunteer in another crisis and beyond.

Acknowledgements Only the authors were involved in the drafting and production of this manuscript.

\section{Declarations}

Conflict of Interest The authors declare no competing interests.

\section{References}

1. Chinsky R, Morris A, Suh A, et al. Medical student perspectives on their role as emerging physicians during the COVID-19 pandemic. Med Sci Educ. 2021;1-9. https://doi.org/10.1007/ s40670-021-01374-z.

2. Lane A, McGrath J, Cleary E, Guerandel A, Malone KM. Worried, weary and worn out: mixed-method study of stress and well-being in final-year medical students. BMJ Open. 2020;10(12):e040245. https://doi.org/10.1136/bmjopen-2020-040245.

3. Jennings ML. Medical student burnout: interdisciplinary exploration and analysis. J Med Humanit. 2009;30(4):253-69. https://doi. org/10.1007/s10912-009-9093-5.

4. Isaac V, McLachlan CS, Walters L, Greenhill J. Screening for burn-out in Australian medical students undertaking a rural clinical placement. BMJ Open. 2019;9(7):e029029. https://doi.org/10. 1136/bmjopen-2019-029029.

5. Torppa MA, Makkonen E, Mårtenson C, Pitkälä KH. A qualitative analysis of student Balint groups in medical education: contexts and triggers of case presentations and discussion themes. Patient Educ Counsel. 2008;72(1):5-11. https://doi.org/10.1016/j.pec. 2008.01.012.

Publisher's Note Springer Nature remains neutral with regard to jurisdictional claims in published maps and institutional affiliations. 